\title{
Micro-CT 3D reconstructions reveal coral growth pattern and parameter
}

Yixin $\mathrm{Li}^{1}$, Tingyu $\mathrm{Han}^{1}$, Kun Bi ${ }^{1}$, J.-Y. Chen ${ }^{2}$, Jing $\mathrm{Lu}^{3}$, Chunpeng $\mathrm{He}^{4}$, and Zuhong $\mathrm{Lu}^{1}$

${ }^{1}$ State Key Laboratory of Bioelectronics

${ }^{2}$ Chinese Academy of Sciences

${ }^{3}$ Key Laboratory of Vertebrate Evolution and Human Origins of Chinese Academy of Sciences

${ }^{4}$ Southeast University

June 16, 2021

\begin{abstract}
Colony is the basic geometric construction of coral reef. However, both the forming regulations of colony and reef are still obscure. In this study, we reconstructed 21 important samples by high-resolution micro-computed tomography to investigate coral growth pattern and parameter. Our skeleton and canal reconstructions revealed characteristics of different coral species and we further visualized the growth axes and growth rings to reveal coral growth directions. We drew a skeleton gray-scale map and calculated coral skeleton void ratios to reveal skeletal diversity, devising a method to quantify coral growth. On the bases of the 3D reconstructions and growth parameters, we investigated growth strategies of different coral species. This work extends the knowledge of how reef-building corals grow their colonies, giving a hint on reef forming regulation. The data of this paper contain large amount of coral growth information, and can be used in further research on reef forming patterns under different conditions. The method used in this study can also be applied in animals with porous skeletons.
\end{abstract}

\section{Hosted file}

Manuscript.docx available at https://authorea.com/users/420011/articles/526391-micro-ct-3dreconstructions-reveal-coral-growth-pattern-and-parameter

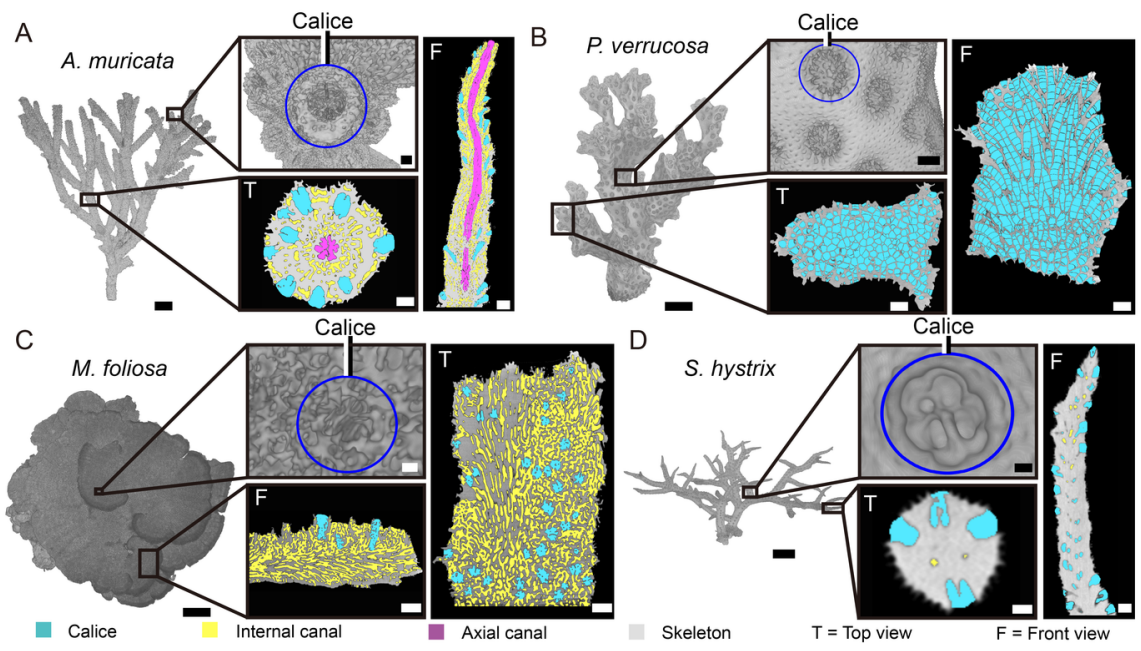




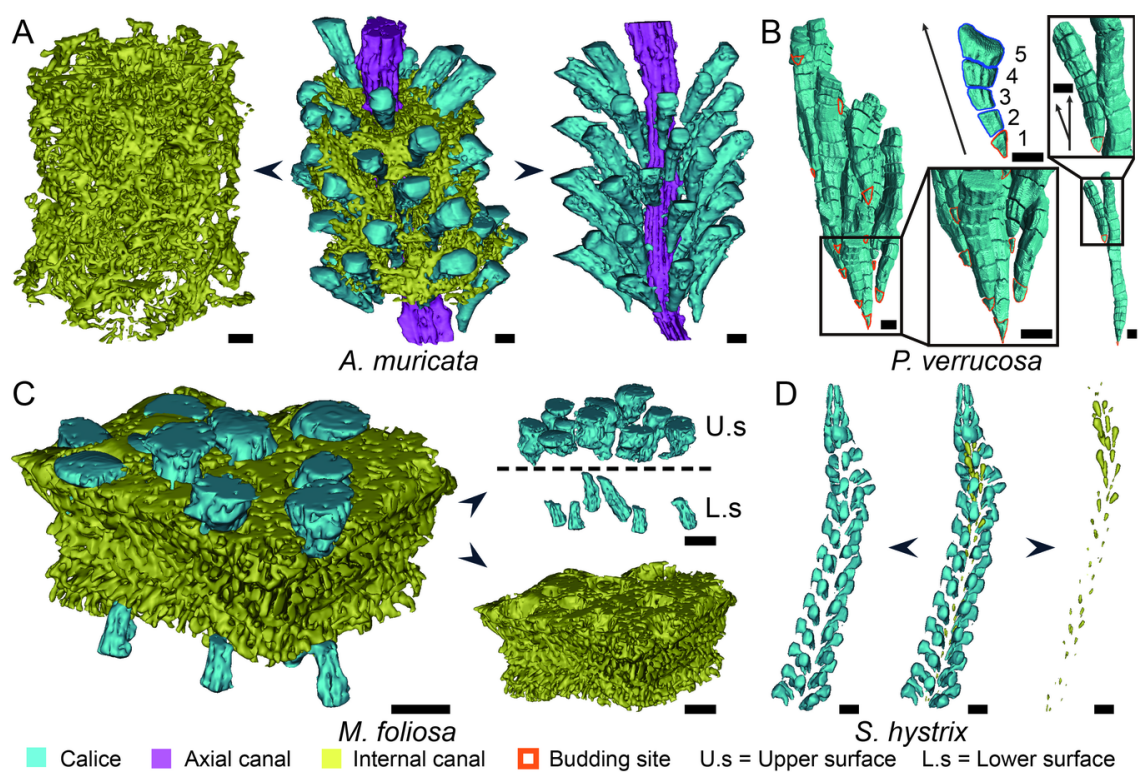




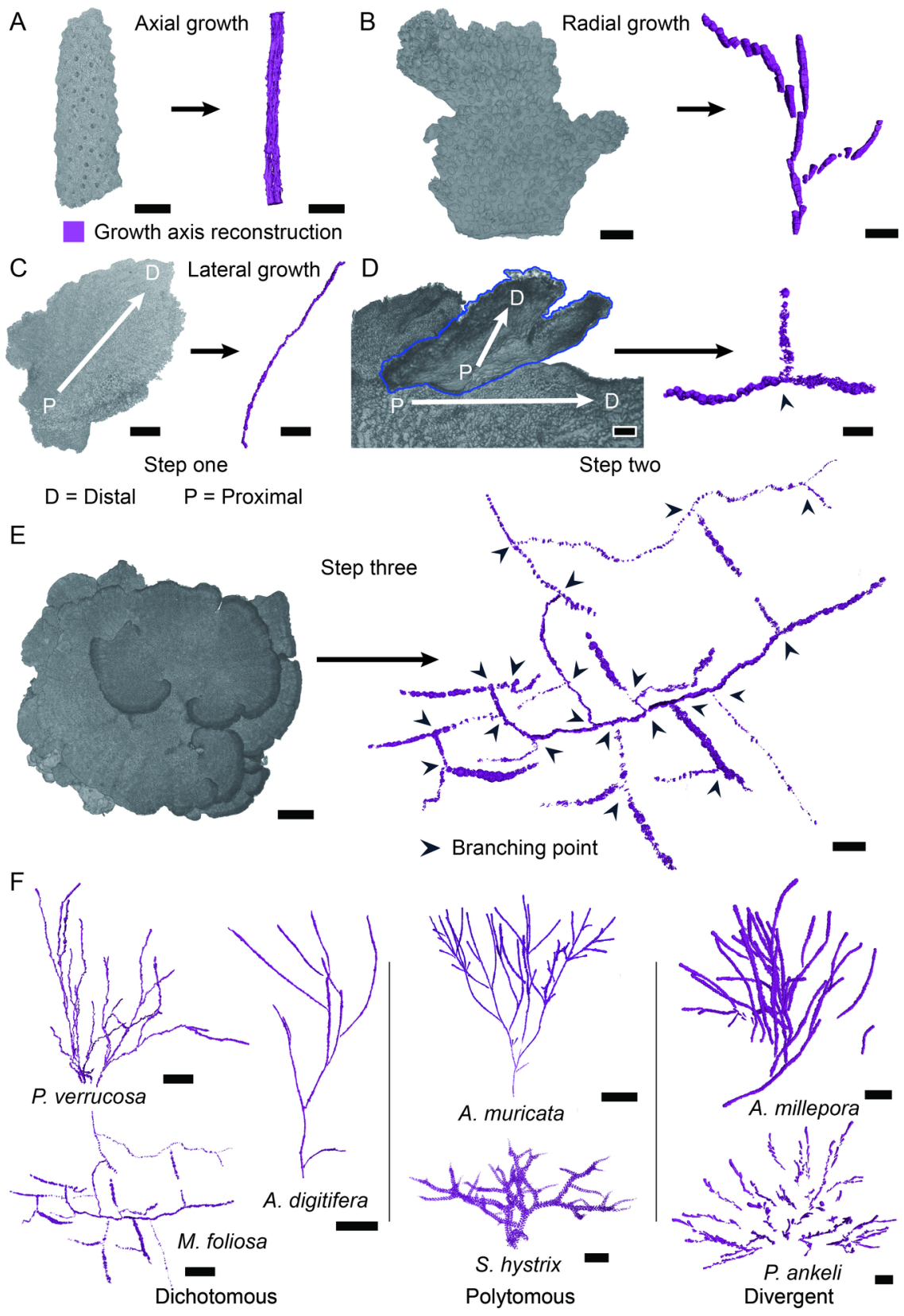




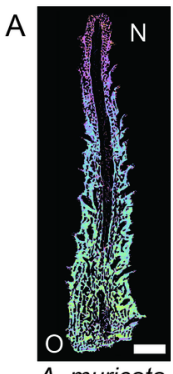

A. muricata

EA Ratio (\%)

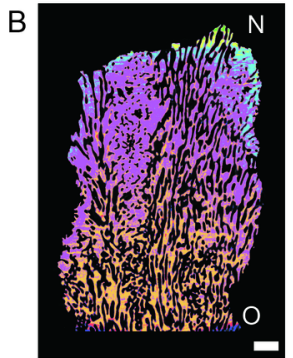

M. foliosa

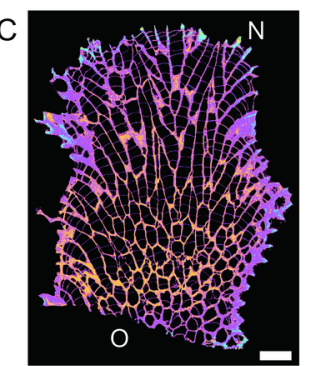

$P$. verrucosa

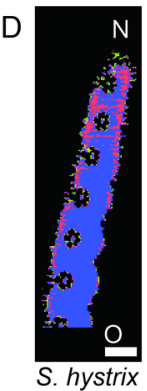

Gray-scale gradient

65000

60000

55000

50000

45000

40000

35000

30000

25000

20000

S. hystrix Species

$P$. verrucosa

M. foliosa

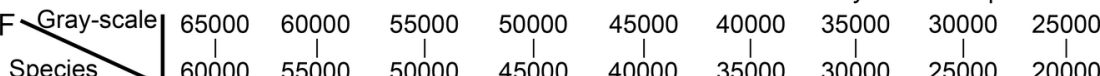
\begin{tabular}{l|ccccccccc}
\hline A. muricata & $0.835 \%$ & $14.333 \%$ & $42.140 \%$ & $20.635 \%$ & $6.046 \%$ & $4.255 \%$ & $4.441 \%$ & $7.315 \%$ & $0.000 \%$ \\
\hline
\end{tabular} \begin{tabular}{l|llllllllll}
$M$. foliosa & $0.070 \%$ & $0.305 \%$ & $3.039 \%$ & $34.840 \%$ & $30.607 \%$ & $11.349 \%$ & $9.629 \%$ & $10.161 \%$ & $0.000 \%$ \\
\hline
\end{tabular}

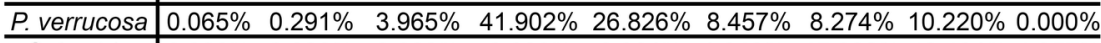

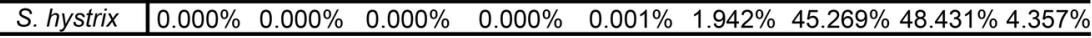

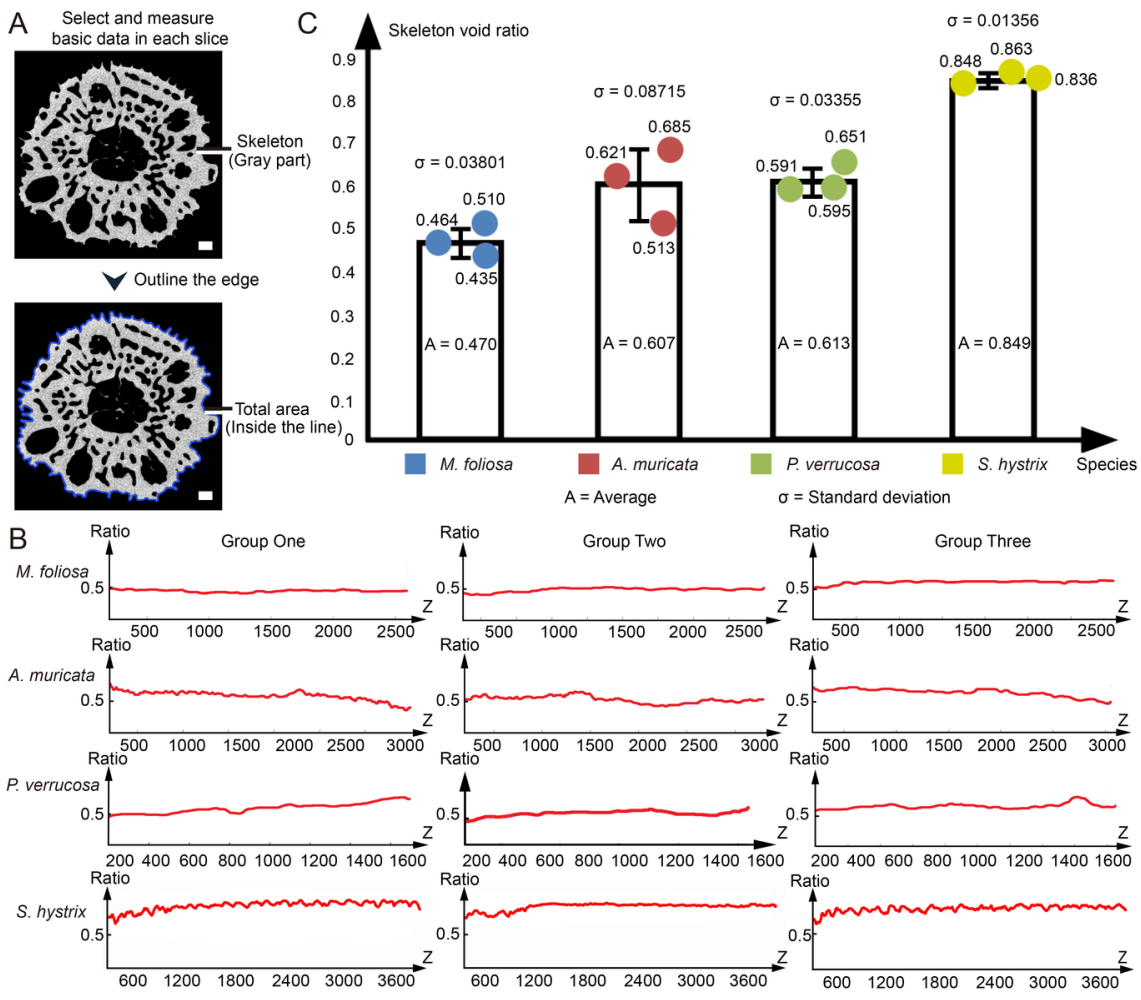




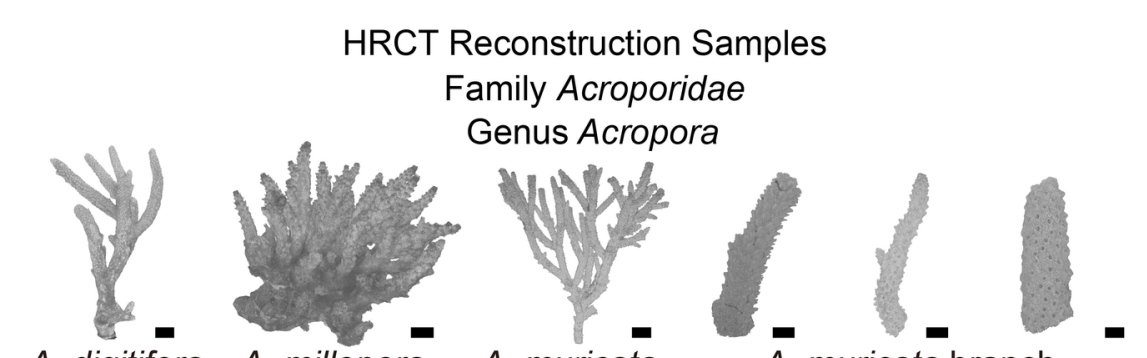

A. digitifera A. millepora A. muricata

A. muricata branch

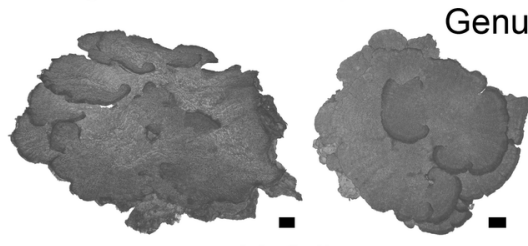

M. foliosa

Genus Montipora

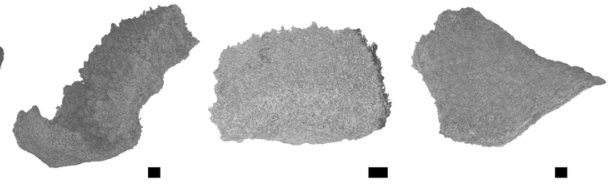

M. foliosa branch

Family Pocilloporidae

Genus Pocillopora

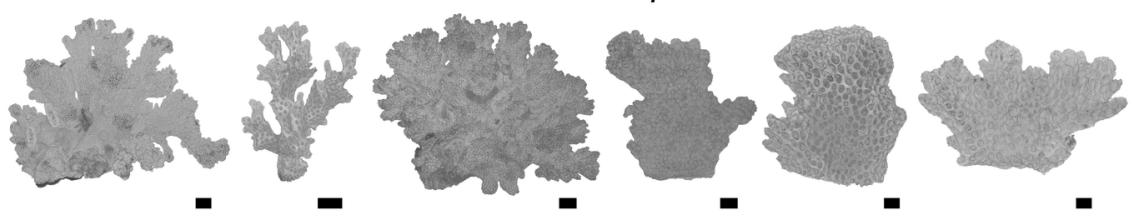

$P$. verrucosa $\quad P$. ankeli $\quad P$. verrucosa branch

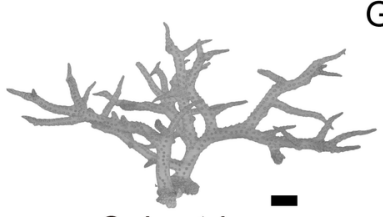

S. hystrix Genus Seriatopora

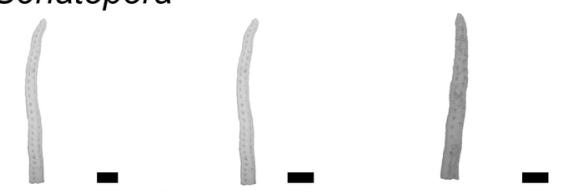

S. hystrix branch

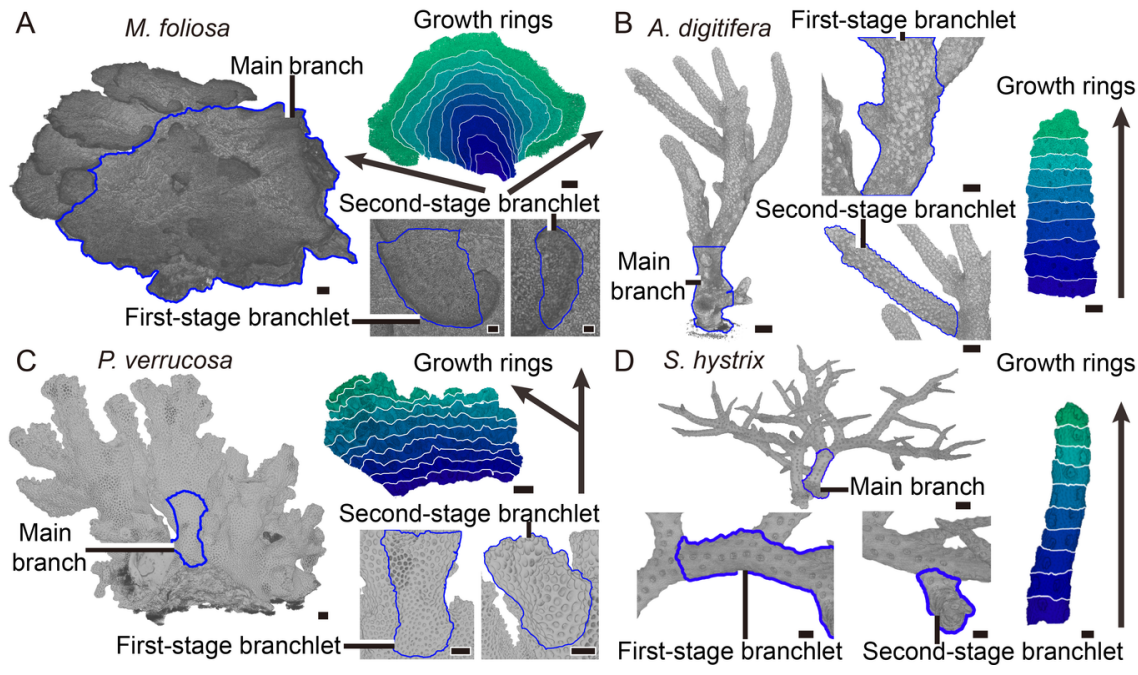

